\title{
Sum and Difference Squeeze Properties of Entangle Coherent States
}

\author{
Yongxin Zhan \\ Department of physics, Sichuan Agriculture university, Sichuan, Ya An, 625014, China \\ Tel: 86-835-288-5166 E-mail: zyx073515@163.com
}

By the national natural science foundation in key projects funded (60601005)

\begin{abstract}
A kind of entangle coherent states is constructed. According to dual mode field's sum and difference operators defined by Mark Hillery, its squeezing properties are investigated by means of numerical calculation. Results show that the states appear sum and difference squeezing effect under the given conditions.
\end{abstract}

Key words: Entangle coherent, Sum operator, Different operator, Quantum optics

\section{Introduction}

The quantum entangle is a basic characteristics different with non-classical physics in quantum mechanics. Since entangle states have been put forward by Einstein, Podolsky(Einstein A, Podolsky B, Rosen N. 1935), Rosen and Schrödinger(Schrödinger E. 1935) to verify completeness of quantum mechanics, they have been attracting extensive attention in physical field. Recently, following the development of quantum information, the entangle states are considered as a kind of important physical resource. Up to now, they have been applied in the region such as quantum computation, teleportation (Bennett C H, Brassard G, Crepeau C, et al. 1993), dense coding(Bennett C H, Wiesner S J. 1992) and quantum key distribution(Ekert A K. 1991).

The research on non-classical effects of optical field is one of important subject in quantum optics. Squeezing effects of optical field reflects a kind of non-classic properties. They have extensive application in optical communication, high precision measurement and weak signal detection (Loudon R, Knight P L. 1987)(Wall D F. 1983). They have become focus in quantum optics.

In the paper (Zhou Lan, Kuang Leman. 2002), entangle degree, dual and single mode squeezing of entangle coherent states have studied and their relations have been discussed. In the paper (Fu H C, Wang X G, Solomon A I. 2001), the relations of entangle degree,squeezing and ant-bunching of entangle coherent states have investigated. In the paper, sum and difference squeeze effects of entangle coherent states are discussed by using sum and difference operator introduced by Mark Hillery (Hillery M. 1989).

\section{Entangle coherent states}

According to paper (Mann A, Sanders B C, Munro W J. 1995)(Wang Xiao-guang. 2002), a kind of entangle coherent states is considered

$$
\mid \psi>=\frac{1}{N}\left[\mu\left|\alpha, \alpha>+v e^{i \phi}\right|-\alpha, \alpha^{*}>\right],
$$

where $\mu$ and $v$ are complex constants, $\alpha=r e^{i \delta}, \alpha^{*}$ is complex conjugation, $|\alpha, \alpha\rangle=|\alpha\rangle \otimes|\alpha\rangle$ and $\left.\left|-\alpha, \quad \alpha^{*}\right\rangle=|-\alpha\rangle \otimes \alpha^{*}\right\rangle, \mid \alpha>$ is Glauber coherent states, $\phi$ is the phase difference between $\mu$ and $v, N$ is normalized coefficient. For convenience, $\mu$ and $v$ are assumed real. Using complete property, $N$ can read

$$
N=\left\{\mu^{2}+v^{2}+2 \mu \nu \cos \left(r^{2} \sin 2 \varphi-\phi\right) \exp \left(-3 r^{2}+r^{2} \cos 2 \varphi\right)\right\}^{-1 / 2} .
$$

For convenience, the first and second mode of dual mode coherent states (1) are notated as $a$ and $b$, respectively.

\section{Sum and difference squeezing of entangle coherent states}

\subsection{Sum squeezing of entangle coherent states}

By the paper (Hillery M. 1989), two orthogonal hermite operators are introduced 
$V_{1}=\frac{a b+a^{+} b^{+}}{2}, V_{2}=\frac{a b-a^{+} b^{+}}{2}$,

Where $a, a^{+}, b$ and $b^{+}$are annihilation and creation operator of $a$ and $b$ mode of dual mode radiation field, respectively. They satisfy with

$$
\begin{aligned}
& {\left[V_{1}, V_{2}\right]=i \frac{a^{+} a+b^{+} b+1}{2},} \\
& <\left(\Delta V_{1}\right)^{2}>\cdot<\left(\Delta V_{2}\right)^{2}>\geq \frac{1}{4}\left|\left[V_{1}, V_{2}\right]\right|^{2},
\end{aligned}
$$

If the inequality is right

$$
<\left(\Delta V_{i}\right)^{2}><\frac{1}{2}\left|\left[V_{1}, V_{2}\right]\right|, \quad(i=1,2)
$$

there is squeeze effect in optical field component $V_{i}(i=1,2)$. With the view of description degree of squeeze, squeezed degree $S_{i}$ is defined

$$
S_{i}=<\left(\Delta V_{1}\right)^{2}>-\frac{1}{2}\left|\left[V_{1}, V_{2}\right]\right| . \quad(i=1,2)
$$

If $S_{i}<0$, it indicates that there is squeezing effect in the component $V_{i}(i=1,2)$.

From the expression (7), the squeezing degrees $S_{i}$ can read

$$
\begin{aligned}
& S_{1}=\frac{1}{4}\left[<a^{2} b^{2}>+<a^{+2} b^{+2}>+2<a^{+} a b^{+} b>-\left(<a b>+<a^{+} b^{+}>\right)^{2}\right], \\
& S_{2}=\frac{1}{4}\left[<a^{2} b^{2}>+<a^{+2} b^{+2}>-2<a^{+} a b^{+} b>-\left(<a b>-<a^{+} b^{+}>\right)^{2}\right] .
\end{aligned}
$$

In order to get values of (8) and (9), according to (1), the below expressions are obtained

$$
\begin{aligned}
& <a^{2} b^{2}>+<a^{+2} b^{+2}>=\frac{2 r^{4}}{N^{2}}\left\{\mu^{2} \cos 4 \varphi+v^{2}+\mu \nu \exp \left(-3 r^{2}+r^{2} \cos 2 \varphi\right) .\right. \\
& \left.\left[\cos \left(r^{2} \sin 2 \varphi-\phi\right)+\cos \left(r^{2} \sin 2 \varphi-\phi+4 \varphi\right)\right]\right\} \\
& <a b>+<a^{+} b^{+}>=\frac{2 r^{2}}{N^{2}}\left\{\mu^{2} \cos 2 \varphi-v^{2}+\mu \nu \exp \left(-3 r^{2}+r^{2} \cos 2 \varphi\right) .\right. \\
& \left.\left[\cos \left(r^{2} \sin 2 \varphi-\phi+2 \varphi\right)-\cos \left(r^{2} \sin 2 \varphi-\phi\right)\right]\right\} \\
& \quad<a b>-<a^{+} b^{+}>=i \frac{2 r^{2}}{N^{2}}\left\{\mu^{2} \sin 2 \varphi+\mu \nu \exp \left(-3 r^{2}+r^{2} \cos 2 \varphi\right) .\right. \\
& \left.\left[\sin \left(r^{2} \sin 2 \varphi-\phi\right)+\sin \left(r^{2} \sin 2 \varphi-\phi+2 \varphi\right)\right]\right\} \\
& <a^{+} a b^{+} b>=\frac{r^{4}}{N^{2}}\left[\mu^{2}-v^{2}-2 \mu \nu \exp \left(-3 r^{2}+r^{2} \cos 2 \varphi\right) \cos \left(r^{2} \sin 2 \varphi-\phi+2 \varphi\right)\right]
\end{aligned}
$$

After (10)-(13) are put into (8) and (9), the sum squeezing properties of entangle coherent state are investigated by means of numerical calculation technique. Under $\mu, v$ and $r$ given certain value, some of graphs are obtained, which show squeeze degree varying with parameter $\delta$ and $\phi$. 


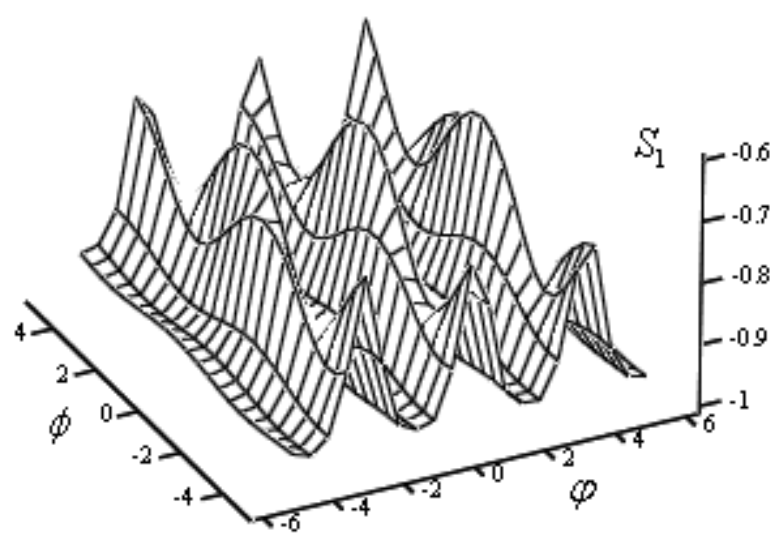

(a)

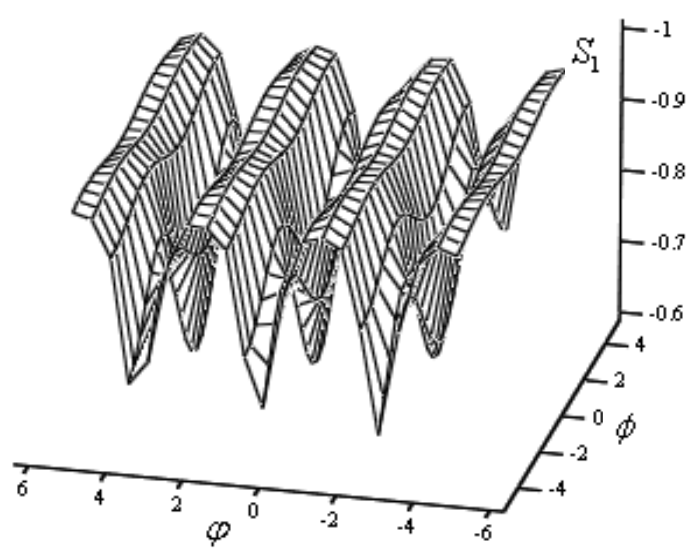

(b)

Figure 1. The sum squeezing degree $\mathrm{S}_{1}$ vary with $\varphi$ and $\phi$ when $\mu=5, v=20, r=1$

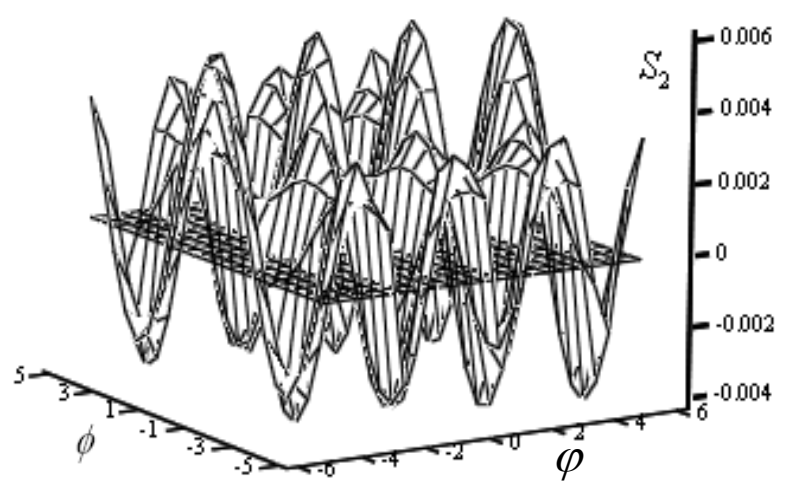

(a)

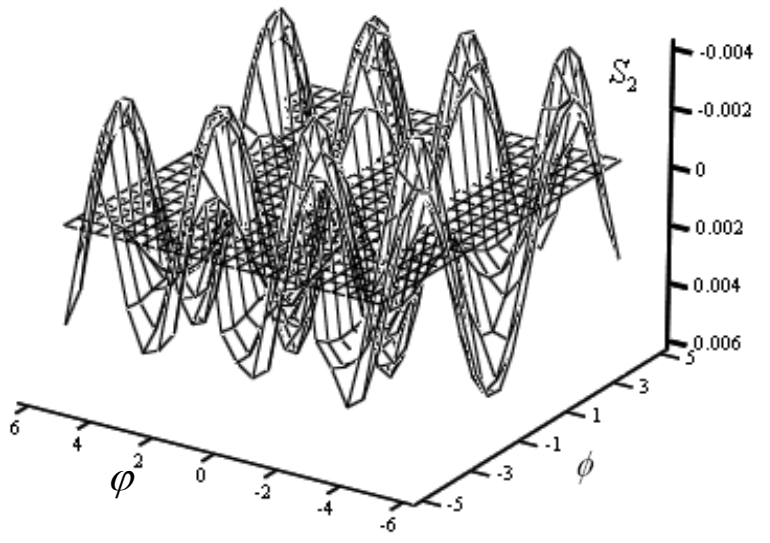

(b)

Figure 2. The sum squeezing degree $\mathrm{S}_{2}$ vary with $\varphi$ and $\phi$ when $\mu=2, v=30, r=0.8$ In fig 1, sub-fig (a) and (b) display that $S_{1}$ varies with $\varphi$ and $\phi$ under $\mu=5, \nu=20$ and $r=1$ from different direction of $S_{1}$ respectively. They show that $S_{1}<0$ in all change range of $\varphi$ and $\phi$ and that $S_{1}$ gets its minimum -0.95 at $\varphi=(2 n+1) \pi / 2$, hence there is squeezing effect in the component $V_{1}$. In fig 2, sub-fig (a) and (b) display that $S_{2}$ varies with $\varphi$ and $\phi$ under $\mu=2, v=30$ and $r=0.8$ from the direction of $S_{2}$. They show that $S_{2}<0$ in some range of $\varphi$ and $\phi$, but squeezing effect is weak in the component $V_{2}$. In addition, $S_{1}$ and $S_{2}$ vary with $\varphi$ and $\phi$ periodically.

3. Difference squeezing of entangle coherent states

Other pair of normal hermite operator is defined

$W_{1}=\frac{a^{+} b+a b^{+}}{2} W_{2}=\frac{a^{+} b-a b^{+}}{2}$,

They satisfy with

$\left[W_{1}, W_{2}\right]=i \frac{a^{+} a-b^{+} b}{2}$, 
$<\left(\Delta W_{1}\right)^{2}>\cdot<\left(\Delta W_{2}\right)^{2}>>\frac{1}{4}\left|\left[W_{1}, W_{2}\right]\right|^{2}$,

If the inequality is right

$<\left(\Delta W_{i}\right)^{2}>\cdot<\frac{1}{2}\left|\left[W_{1}, W_{2}\right]\right| \quad(i=1,2)$

then there is squeeze effect in optical field component ${ }^{W_{i}}$. With the view of description degree of squeeze, squeezed degree $S_{i}$ is defined

$S_{i}=<\left(\Delta W_{1}\right)^{2}>-\frac{1}{2}\left|\left[W_{1}, W_{2}\right]\right| \quad(i=1,3)$

From the expression (17), the squeezing degrees $S_{i}$ can read

$$
\begin{aligned}
& S_{3}=\frac{1}{4}\left[<a^{+2} b^{2}>+<a^{2} b^{+2}>+2<a^{+} a b^{+} b>+2<b^{+} b>-\left(<a^{+} b>+<a b^{+}>\right)^{2}\right] \\
& S_{4}=\frac{1}{4}\left[<a^{+2} b^{2}>+<a^{2} b^{+2}>-2<a^{+} a b^{+} b>-2<b^{+} b>-\left(<a^{+} b>-<a b^{+}>\right)^{2}\right]
\end{aligned}
$$

In order to obtain value of (19) and (20), according to (1), the below expressions are got

$$
\begin{aligned}
& <a^{+2} b^{2}>+<a^{2} b^{+2}>=\frac{2 r^{4}}{N^{2}}\left\{\mu^{2}+v^{2} \cos 4 \delta+\mu \nu \exp \left(-3 r^{2}+r^{2} \cos 2 \delta\right) .\right. \\
& \left.\left[\cos \left(r^{2} \sin 2 \delta-\phi+4 \delta\right)+\cos \left(r^{2} \sin 2 \delta-\phi\right)\right]\right\} \\
& <b^{+} b>=\frac{r^{2}}{N^{2}}\left\{\mu^{2}+v^{2}+2 \mu \nu \exp \left(-3 r^{2}+r^{2} \cos 2 \varphi\right) \cos \left(r^{2} \sin 2 \delta-\phi\right)\right\} \\
& <a^{+} b>+<a b^{+}>=\frac{2 r^{2}}{N^{2}}\left\{\mu^{2}-v^{2} \cos 2 \delta+\mu \nu \exp \left(-3 r^{2}+r^{2} \cos 2 \varphi\right) .\right. \\
& \left.\left[\cos \left(r^{2} \sin 2 \delta-\phi+2 \delta\right)-\cos \left(r^{2} \sin 2 \delta-\phi\right)\right]\right\} \\
& <a^{+} b>-<a b^{+}>=\frac{i 2 r^{2}}{N^{2}}\left\{v^{2} \sin 2 \delta-\mu \nu \exp \left(-3 r^{2}+r^{2} \cos 2 \varphi\right) .\right. \\
& \left.\left[\sin \left(r^{2} \sin 2 \delta-\phi\right)+\sin \left(r^{2} \sin 2 \delta-\phi+2 \delta\right)\right]\right\}
\end{aligned}
$$

After (13), (21)-(24) are put into (19) and (20), the difference squeezing properties of entangle coherent state are investigated by means of numerical calculation technique. Under $\mu, v$ and $r$ given certain value, some of graphs are obtained, which show squeeze degree varying with parameter $\delta$ and $\phi$. 


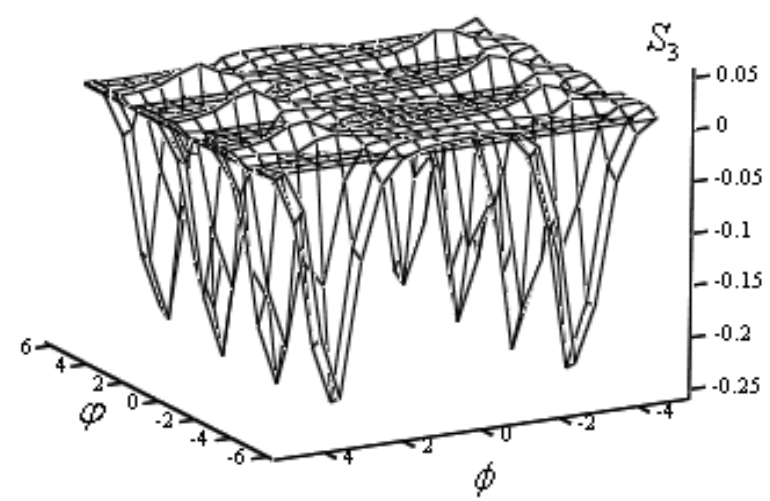

(a)

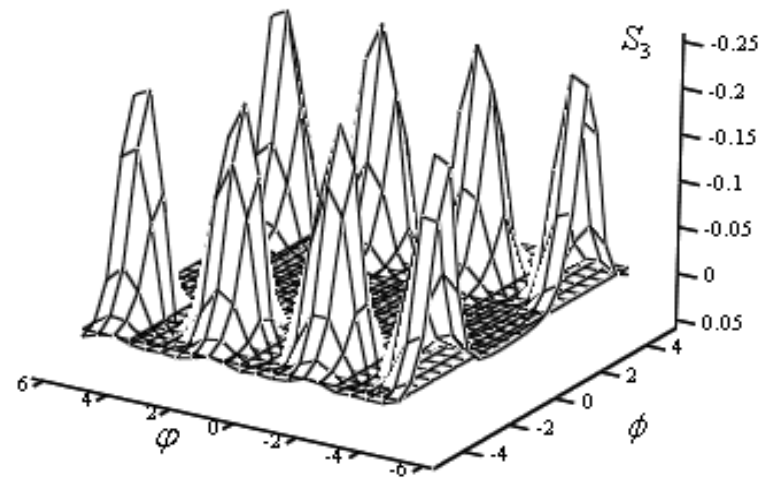

(b)

Figure 3. The sum squeezing degree $\mathrm{S}_{3}$ vary with $\varphi$ and $\phi$ when $\mu=8, \nu=8, r=0.3$

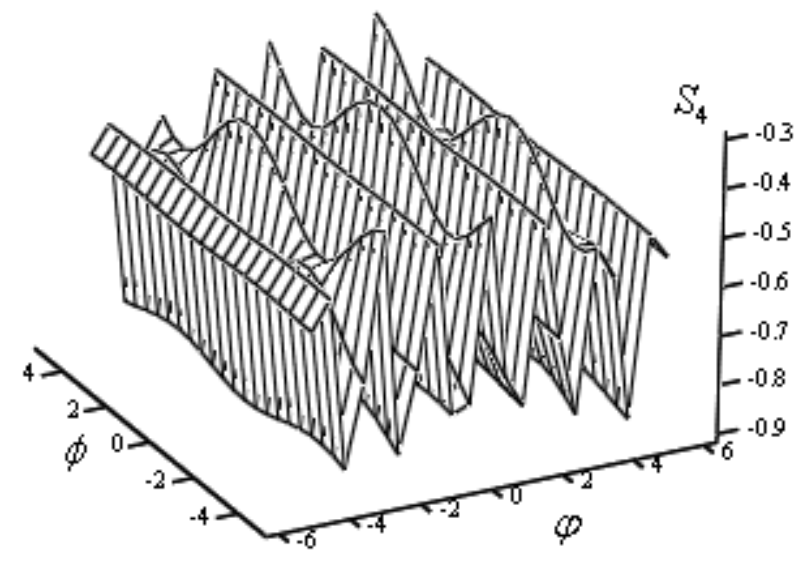

(a)

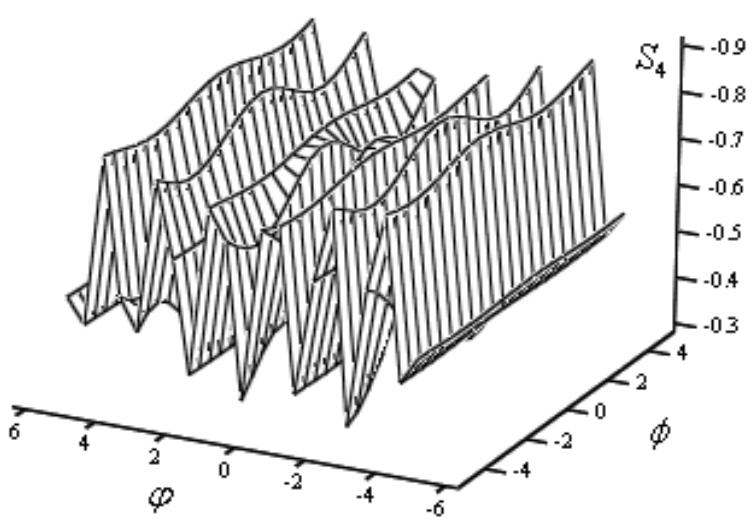

(b)

Figure 4. The sum squeezing degree $\mathrm{S}_{4}$ vary with $\varphi$ and $\phi$ when $\mu=20, v=30, r=1.2$

In figure 3, sub-figure (a) and (b) display that $S_{3}$ varies with $\varphi$ and $\phi$ under $\mu=8, v=8$ and $r=0.3$ from different direction of $S_{3}$, respectively. They show that $S_{3}<0$ in some change range of $\varphi$ and $\phi$, hence there is squeezing effect in the component $W_{1}$. In fig 4, sub-figure (a) and (b) display that $S_{4}$ varies with $\varphi$ and $\phi$ under $\mu=20, v=30$ and $r=0.8$ from different direction of $S_{4}$, respectively. They show that $S_{4}<0$ in all change range of $\varphi$ and $\phi$, therefore there is squeezing effect in the component $W_{2}$. And meantime $S_{3}$ and $S_{4}$ vary with $\varphi$ and $\phi$ periodically.

\section{Conclusions}

In the paper, a kind of entangle coherent states is introduced. According to the concepts of sum and difference operator introduced by Mark Hillery about two mode radiation field, by means of using numerical theology, non-classical properties of entangle coherent states are investigated. Results show that the entangle coherent states may appear sum and different squeezing effect under given conditions.

\section{References}

Bennett C H, Brassard G, Crepeau C, et al. (1993). Teleporting an unknown quantum state via dual classical and Einstein-podolsky-Rosen channels. Phys Rev Lett, 1993, 70(13): 1895-1899.

Bennett C H, Wiesner S J. (1992). Communication via one-and-two particle operators on Einstein-podolsky-Rosen states. Phys Rev Lett, 1992, 69(20): 2881-2884. 
Einstein A, Podolsky B, Rosen N. (1935). Ca quantum-mechanical description of physical reality be considered complete. Phys Rev, 1935, 47(10): 777-780.

Ekert A K. (1991). quantum cryptography based on bell's theorem. Phys Rev Lett, 1991,661-663.

Fu H C, Wang X G, Solomon A I. (2001). Maximal entanglement of nonorthogonal states: classification. Phys Lett A, 2001,291: 73.

Hillery M. (1989). Sum and different squeezing of the electromagnetic field. Phys Rev A 1989, 40(6): 3147-3155.

Loudon R, Knight P L. (1987). Squeezed light. J Mod Opt, 1987, 34(6-7): 709-759

Mann A, Sanders B C, Munro W J. (1995). Bell's inequality for an entanglement of non-orthogonal states. Phys $\operatorname{Rev} A, 1995$, 51(2): 989-991.

Schrödinger E. (1935). Die gegenwartige situation in der quantum chanik. Naturwissenschaften, 1935, 23: 807-812, 823-828, 844-849.

Wall D F. (1983). Squeezed states of light. Nature, 1983, 306(10): 141-146.

Wang Xiao-guang. (2002). Bipartite entangled non-orthogonal states. J Phys A: Math Cen,2002,35:165-173.

Xia Y J, Gao D Y. (2007). Entangle coherent states and their nonclassical effects. Acta Phys. Sin. 2007, 56(7): 3703-3708.

Zhou Lan, Kuang Leman. (2002). Optical preparation of entangled squeezed vacuum states. Phys Lett A, 2002, 302: 273. 